\title{
Cellular Therapy Targeting Functional Outcome in a Case of Cervical Spinal Cord Injury
}

\author{
Alok Sharma ${ }^{1}$, Hemangi Sane ${ }^{2}$, Dipti Khopkar ${ }^{3}$, Nandini Gokulchandran $^{4}$, Hema Biju, \\ V C Jacob ${ }^{6}$ and Prerna Badhe ${ }^{7}$ \\ ${ }^{1,4,7}$ Department of Medical Services and Clinical Research, NeuroGen Brain and Spine Institute, Surana \\ Sethia Hospital and Research Centre, Suman Nagar, Sion, Mumbai, India \\ ${ }^{2,3}$ Department of Research and Development, NeuroGen Brain and Spine Institute, Surana Sethia \\ Hospital and Research Centre, Suman Nagar, Sion, Mumbai, India \\ ${ }^{5,6}$ Department of Neurorehabilitation, NeuroGen Brain and Spine Institute, Surana Sethia Hospital and \\ Research Centre, Suman Nagar, Sion, Mumbai, India
}

Correspondence should be addressed to: Dipti Khopkar; diptikhopkar@gmail.com

Received date: 20 January 2014; Accepted date: 12 March 2014; Published date: 11 June 2014

Academic Editor: Biju George

Copyright (C) 2014. Alok Sharma, Hemangi Sane, Dipti Khopkar, Nandini Gokulchandran, Hema Biju, V C Jacob and Prerna Badhe. Distributed under Creative Commons CC-BY 3

\begin{abstract}
Traumatic Spinal Cord Injury is a neurological condition characterized by partial or complete sensory/motor loss below the level of lesion. Definitive treatment directed towards repair, regeneration of the severed cord has not been established. The recovery of these patients is quite slow and in some cases it attains a plateau. Here we present a case report of a 23 year old male with traumatic quadriplegia at $\mathrm{C}_{6}$ level. He was administered autologous bone marrow mononuclear cells intrathecally, followed by intensive neurorehabilitation. Prior to this intervention, the patient was assessed on Functional Independence Measure (FIM) and other neurological assessments. He scored 58/126 on FIM and exhibited weakness on the left side and loss of sensations on the right. He was completely dependent on all his Activities of Daily Living (ADL's) except for communication and social cognition. Six months post cellular therapy, there was improvement in thefunctional status with FIM score $76 / 126$. He improved in bed mobility, static and dynamic balance, transfers and ADL's. He was able to walk with walker and AFO with push knee splint on left independently. His vocational status also improved. This case report contributes to the literature as one of the preliminary evidence suggesting that cell therapy along with neurorehabilitation may fasten the recovery processes in patients with traumatic spinal cord injuries.
\end{abstract}

Keywords: cervical spine, spinal cord injury, cell therapy, bone marrow, rehabilitation.

Cite this Article as: Alok Sharma, Hemangi Sane, Dipti Khopkar, Nandini Gokulchandran, Hema Biju, V C Jacob and Prerna Badhe (2014)," Cellular Therapy Targeting Functional Outcome in a Case of Cervical Spinal Cord Injury", Advances in Stem Cells, Vol. 2014 (2014), Article ID 149752, DOI: 10.5171/2014.149752 


\section{Introduction}

Traumatic spinal cord injuries results in motor and/ or sensory loss below the level of injury. Functionally, they affect the activities of daily living, work and leisure activity. Thus, the quality of life is compromised. The recovery after spinal cord injury is dependent on factors like the level and extent of injury, sensory-motor preservation below level of injury and rehabilitation. In an incomplete spinal cord injury, some amount of information can still pass through the site of injury, but is fragmented or distorted as discussed by Rainteau et al (2001).

In the area of rehabilitation, there are few approaches which are directed towards improving the functional status of these patients (Granat et al 1993; Behrman et al 2000). Though the scientific understanding of Central Nervous System (CNS) regeneration has advanced greatly in the past twenty years, there are still many unknowns with respect to inducing successful regeneration (Fiblin 2003; Houle et al 2003; Hulsebosch 2002; Kwon et al 2002). Autologous bone marrow mononuclear cells have received attention for transplantation in spinal cord injuries. Studies have explored these cells as a viable, safe and easily accessible treatment option to facilitate physiological, structural, neurological and functional recovery in patients with spinal cord injuries by processes of neurorestoration. This in turn enhances repair of neural tissue at the site of injury as seen in the studies of Satake et al 2004; Chopp et al 2000 and Murray et al 2002.

Presented herewith, is a case report of a 23 year old male who suffered from traumatic $\mathrm{C}_{6}$ quadriplegia, and was treated with the use of cell therapy in addition to standard neurorehabilitation.

\section{Case Report}

A 23 year old male suffered a road traffic accident leading to spinal cord injury and quadriplegia. The immediate CT (Computerised Tomography) scan of spine showed severe compression/burst fracture of the $\mathrm{C}_{6}$ vertebral body. MRI (Magnetic Resonance Imaging) revealed acute mild compression fracture of the C6 vertebral body, spinal cord contusion and small traumatic postero-central protrusion of C5-6 disc that was indenting the thecal sac. He was conservatively managed with medications, traction, cervical collar and physiotherapy for 6 months. Since the sensorimotor recovery was quite slow and insignificant functionally, the patient decided to undergo cellular therapy.

On examination prior to cellular therapy, hypertonia and hyper reflexia with grade 2 spasticity on Modified Ashworth Scale (MAS) was recorded. On Manual Muscle Testing (MMT), the patient scored grade 0 in left lower limb and grade 3 in the right lower limb. Abdominals and back extensors were grade 2 , whereas proximally both the upper limbs had strength of grade 2 and grade 0 distally. He had partial sensory recovery below the D4 level. Sensations were better in the left half of the body, whereas motor recovery was seen more on the right side. He had complete loss of bowel and bladder control and was on condom catheter for the same. Hand functions were poor. Static balance in sitting was fair, whereas dynamic balance was poor as he was unable to perform any activities when made to sit. Functionally, he was wheelchair bound for mobility. He was completely dependent on his caregiver for performing functions like grooming, bathing, upper and lower body dressing and bowel/bladder management. He needed maximum assistance in eating and toileting, moderate assistance in mobility and transfers in bed and toileting. He was completely independent in the areas of communication and social cognition. On Functional Independence Measure (FIM), he scored 58/126.

After a detailed pre-assessment, the patient underwent autologous bone marrow 
mononuclear cells (BMMNCs) transplantation. The procedure was a part of the open label study. A formal approval from ICSRT (Institutional Committee for Stem Cell Research and Therapy) standard informed consent was taken prior to the procedure from the patient and the family. Before the aspiration of bone marrow, the patient is subjected to the following tests like complete blood count, blood sugar, serum creatinine, serum electrolytes, liver function test, clotting time, bleeding time, prothrombin time, Human Immunodeficiency Virus I and II antibodies, Hepatitis B surface Antigen (HbsAg), Hepatitis C Virus antibodies (HCV) and urine routine. Only after the normal test reports, bone marrow is aspirated and collected under sterile aseptic conditions in the operation theatre. With the patient in supine position, local anesthesia was given in the region of anterior superior iliac spine. Using a bone marrow aspiration needle, $100 \mathrm{ml}$ of bone marrow was aspirated and collected in heparinized tubes and transported using a sterile transporter kit immediately to a sterile laboratory, where aerobic, anaerobic and fungal cultures are performed. In the laboratory, the MNCs were separated by the density gradient method. The cells were sent for CD34 counts and viability. The total number of MNCs was $5.7 \times$ $10^{7}$ out of which $96 \%$ were viable. The CD34+ dose was $1.10 \%$. The cells were then transported back to the OT in a sterile cool container. The patient was put in a left lateral position and using a lumbar puncture needle and CSF drainage set, the thecal sac was punctured in the L4-L5 space. Injection at cervical level is associated with major complications of injury to the spinal cord. Therefore, we injected at L4-L5 level which is a safe level as the spinal cord ends at L1 level. A catheter was introduced into the thecal sac and the cells were injected through the catheter. The catheter was withdrawn after the cells were injected. Methylprednisolone $1 \mathrm{gm}$ in $500 \mathrm{ml}$ Ringer Lactate was given intravenously, simultaneously during the injection over a period of hours, as transplantation of cells into the CSF may cause a local inflammatory reaction. MNCs were also injected at specific motor points bilaterally intramuscularly in the following muscles: lumbricals, opponens, abductor digiti minimi, adductor pollicis, interossei, abdominals and also in the left triceps.

Following the procedure, the patient did not report any side effects. The patient then underwent standard neurorehabilitation which included physiotherapy, occupational therapy and urorehabilitation.

At one week there was some improvement in the trunk muscle strength, which enabled him to come supine to sit independently. The strength of triceps had increased as he was able to bend his left elbow and maintain it stable while lying. His pinch strength had improved as he was able to hold a spoon and eat by himself with right hand and able to brush by himself. He had started to use his right upper extremity more purposefully, like, wearing a $\mathrm{T}$ shirt and lower body dressing. He was able to stand with the help of left push knee splint and walker without any assistance. He could maintain standing without assistance for 4-5 minutes and could manage to take 3-4 steps. His sitting balance at the edge of bed had improved. His overall FIM score increased from 58 to 66/126.

The patient continued the rehabilitation at home and followed up 6 months after the discharge. Detailed reassessment was done. Previous improvements seen were maintained, along with further changes. He showed improved strength on the right side in both upper and lower limbs. Strength also improved on the right side in the interossei, lumbricals, abductor pollicis longus and brevis. He was able to shift himself on the edge of the bed. His ADL activities also improved, mainly brushing and was able to wash his face independently. Independent dressing of the upper body like wearing $t$ shirt was achieved. He required assistance while bathing, but he is able to wash his hair independently. He could walk independently with the help of a walker and use of AFO and push knee splint on left. He showed left lower extremity dragging along with hip hiking. 
The patient also reported improved transfers from chair to bed independently. Vocationally, he started going to his brother's gaming zone. He does promotional activities and works on computer. His right hand precision on mouse has improved. All the above changes lead to an increase in his FIM scores from 66 to 76 (overall from 58 to 76).

\section{Discussion}

The above case represented a 23 year old male with C6 traumatic quadriplegia. He underwent transplantation of autologous BMMNCs followed by intensive neurorehabilitation. The patient continued the rehabilitation at home and followed up after 6 months of the treatment. One week post cell therapy with neurorehabilitation as well during a follow up after 6 months, the patient showed continued clinical improvements in many areas. He improved in his strength, stability, balance, gait, transfers, ADL's and hand functions. He was independent in performing many of the activities which were essential for him and was dependent on his caregiver previously. His overall functional status improved subjectively as well as objectively.

The possible mechanism of action underlying these clinical changes may be the physiological changes happening at the cellular level. Autologous BMMNCs were the choice of cells because of the properties they possess. These MNCs contain hematopoietic cells, tissue-specific progenitor cells, stromal cells, and specialized blood cells in different stages of development. They are multipotent, with definitive in vivo and in vitro neuronal differentiation and no associated immunological or ethical issues. It has been reported by Lawall et al in 2010 that the use of whole BMMNCs is more successful than methods using sub fractionated cell preparations. The MNCs injected home themselves at the site of injury of the spinal cord, where they enhance angiogenesis, which contributes to neovascularisation, by producing signaling molecules such as fibroblast growth factor (FGF2) and vascular endothelial growth factor (VEGF). These cells help in remodeling and preventing apoptosis, along with decreasing inflammation at the site of injury.

There is an expanding literature regarding the effects of cell therapy in various neurological conditions, including spinal cord injury. Symptomatic and functional improvements were seen in patients with spinal cord injury at thoracolumbar and cervical levels, which were analyzed in various studies done to find the clinical effects of cell therapy (Sharma et al 2013; Sharma et al 2013; Sharma et al 2011 and Sharma et al 2012). Transplanted bone marrow cells were found to improve neurologic deficits in CNS injury models by generating neural cells or myelin-producing cells (Chopp et al 2000 and Akiyama et al 2002). There are also few hypotheses which are proposed to explain the role of stem cell in spinal cord injury. Bone marrow cells improve neurological deficit by generating either neural cells or myelin producing cells (Chopp et al 2000 and Sasaki et al 2001).

Another major mechanism hypothesized is that the transplanted bone marrow cells guide axonal regeneration at the site of lesion. And also, these transplanted cells promote compensatory mechanisms in order to reorganize the neural network (Wu et al 2003). It has been observed that, there is a loosening of the neuromuscular junctions as a result of the non-use of muscles. Thus, intramuscular injections were given to repair the neuromuscular connectivity. The rationale behind selecting the muscles was that these muscles were already functioning partially and thus had a better chance to improve.

Superimposed on this cellular therapy was the intensive neurorehabilitation which helped in remodeling the cells at the site of injury and enhancing the functions of these cells. The rehabilitation helped in the formation of neural networks by the process of neuronal sprouting thus improving his functional status (Jacobs et al 2004 and 
Wiskemann et al 2011). It has been observed in the literature, that by 6 months after the injury, glial scar formation already begins, thus the clinical representation of plateau phase (Rowland et al 2008). The patient received cell therapy after 6 months, when he demonstrated slow and insignificant recovery, even with the ongoing rehabilitation. Post cell transplantation, the patient showed faster and functionally significant changes with combination of cell therapy and rehabilitation. Therefore, the recovery seen cannot be attributed to therapy alone. Improved functional status of the patient led to better vocational status. Further follow up of the patient is awaited for analyzing long term effects of this combination of cellular therapy with neurorehabilitation.

The clinical changes recorded in the above case report suggest that autologous BMMNCs

appears to be a promising approach in improving the functional status of patients with spinal cord injury. Though not a cure, it may improve the functional status of this set of patients thus upgrading their quality of life. Further studies with larger and homogenous samples, along with the incorporation of an objective imaging to visualize the changes happening at the site of injury, will strengthen the results obtained in this case report. This report is a primary level of evidence, based on which studies with high level of evidence may be performed.

\section{References}

1. Akiyama, Y., Radtke, C., Kocsis, J. D. (2002) "Remyelination of the rat spinal cord by transplantation of identified bone marrow stromal cells," J. Neurosci, 22(15), 6623-6630.

2. Behrman, A. L., Harkema, S. J. (2000) "Locomotor training after human spinal cord injury: A Series of Case Studies," Phys Ther, 80, 688-700.
3. Chopp, M., Zhang, X. H., Li, Y., Wang, L., Chen, J., Lu, D., et al. (2000) "Spinal cord injury in rat: treatment with bone marrow stromal cell transplantation," Neuroreport.

4. Fiblin, M. T. (2003) "Myelin-associated inhibitors of axonal regeneration in the adult mammalian CNS," Nature Reviews Neurosci, 4, $1-11$.

5. Granat, M. H., Ferguson, A. C. B., Andrews, B. J., Delargy, M. (1993) "The role of functional electrical stimulation in the rehabilitation of patients with incomplete spinal cord injury - observed benefits during gait studies," Paraplegia, 31: 207-215.

6. Houle, J. D., Tessler, A. (2003) "Repair of chronic spinal cord injury," Exp. Neurol, 182, 247-260.

7. Hulsebosch, C. E. (2002) "Recent advances in pathophysiology and treatment of spinal cord injury," Adv. Physiol. Educ, 26, 238-255.

8. Jacobs, P. L., Nash, M. S. (2004) "Exercise Recommendations for Individuals with Spinal Cord Injury," Sports Med, 34(11), 727751.

9. Kwon, B. K., Oxland, T. R., Tetzlaff, W. (2002) "Animal models used in spinal cord regeneration research," Spine 27, 1504-1510.

10. Lawall, H., Bramlage, P., Amann, B. (2010) "Stem cell and progenitor cell therapy in peripheral artery disease. A critical appraisal," Thromb Haemost, 103:696-709.

11. Murray, M., Kim, D., Liu, Y., Tobias, C., Tessler, A., Fischer, I. (2002) "Transplantation of genetically modified cells contributes to repair and recovery from spinal injury," Brain Res Rev, 40, 292-300.

12. Raineteau, O., Schwab, M. E. (2001) "Plasticity of motor systems after incomplete spinal cord injury review," Mc Millan Magazines Ltd 2. 
13. Rowland, J. W., Hawryluk, G. W. J., Kwon, B., Fehlings, M. G. (2008) "Current status of acute spinal cord injury pathophysiology and emerging therapies: promise on the horizon," Neurosurgical Focus, 25(5), E2

14. Sharma, A., Gokulchandran, N., Sane, H., Badhe, P., Kulkarni, P., Lohia, M., et al. (2013) "Detailed analysis of the clinical effects of cell therapy for thoracolumbar spinal cord injury: an original study," Journal of Neurorestoratology, 1, 3-22.

15. Sharma, A., Sane, H., Gokulchandran, N., Kulkarni, P., Thomas, N., Bhovad, P., et al. (2013) "Role of Autologous Bone Marrow Mononuclear Cells in Chronic Cervical Spinal Cord Injury-A Longterm Follow Up Study." J Neurol Disord, 1-4.

16. Sharma, A., Badhe, P., Kulkarni, P., Gokulchandran, N., Chopra, G., Lohia, M., et al. (2011) "Autologous Bone marrow Derived mononuclear cells for the treatment of spinal cord injury," The Journal of Orthopaedic and Trauma surgery 1(1), 33-36.

17. Sharma, A., Gokulchandran, N., Chopra, G., Kulkarni, P., Lohia, M., Badhe, P., et al. (2012) "Administration of autologous bone marrow derived mononuclear cells in children with incurable neurological disorders and injury is safe and improves their quality of life," Cell transplantation 21(1), S79-S70.

18. Satake, K., Lou, J., Lenke, L. G. (2004) "Migration of mesenchymal stem cells through cerebrospinal fluid into injured spinal cord tissue," Spine.

19. Sasaki, M., Honmou, O., Akiyama, Y., Uede, T., Hashi, K., Kocsis, J. D. (2001) "Transplantation of an acutely isolated bone marrow fraction repairs demyelinated adult rat spinal cord axons," Glia, 35(1), 26-34.

20. Wu, S., Suzuki, Y., Ejiri, Y., Noda, T., Bai, H., Kitada, M., et al (2003) "Bone marrow stromal cells enhance differentiation of cocultured neurosphere cells and promote regeneration of injured spinal cord," J. Neurosci. Res 72, 343-351.

21. Wiskemann, J., Dreger, P., Schwerdtfeger, R., Bondong, A., Huber, G., Kleindienst, N., et al (2011) "Effects of a partly selfadministered exercise program before, during, and after allogeneic stem cell transplantation," Blood 117(9). 